\title{
Spectral Development of Picosecond Pulses of Mode-Locked Nd-Glass Lasers
}

\author{
ALFONS PENZKOFER AND NORBERT WEINHARDT
}

\begin{abstract}
The spectra of single picosecond pulses of modetocked $\mathrm{Nd}$-glass lasers are investigated along the pulse train. In addition to self-phase modulation, the spectra are modified due to spectral hole burning in the inhomogeneous gain profile of the active medium.
\end{abstract}

\section{INTRODUCTION}

$\mathbf{M}$ ODE-locked $\mathrm{Nd}$-glass lasers generate bandwidth limited picosecond light pulses only in the early part of the pulse train [1]-[8]. Towards the maximum of the pulse train, the spectra broaden and irregular spectra are observed beyond it [1]-[11]. Only in a few reports was small spectral broadening over the whole pulse train in Nd-phosphate glass lasers reported [12], [13]. Self-phase modulation is thought to cause the spectral broadening and structuring [1]-[13]. Enhancement of self-phase modulation and corresponding spectral broadening by self-focusing is discussed in [3], [14]. The influence of nonlinear dispersion, which modulates the temporal pulse shape and subsequently increases the self-phase modulation, is analyzed in [15].

In this paper, we present experimental data which indicate that in addition to self-phase modulation, the spectra are structured and the frequency is shifted by spectral hole burning in the inhomogeneous gain profile of the active medium in the saturation region [16]. We studied the spectral development of picosecond light pulses along the pulse train for passively mode-locked Nd-phosphate, Nd-fluorophosphate, and Ndsilicate glass lasers. Bandwidth-limited pulses are only obtained in the early part of the pulse train. Towards the maximum of the pulse train, the spectra broaden and begin to be modulated due to self-phase modulation. Beyond the pulse train maximum, the peak emission at the central frequency reduces or diminishes and new, spectrally small peaks build up on the high-frequency side and towards the end of the pulse train at the low-frequency side of the central frequency.

The spectral shape in the trailing part of the pulse trains is thought to be a result of gain depletion at the center frequency while high gain is acting in the spectral wings during the opening time of the mode-locking dye.

The occurrence of remarkable self-focusing is hindered by two-photon absorption in the Nd-glass rod [17], which limits the peak pulse intensity in the oscillator [18].

\section{EXPERIMENTAL}

The experimental system is depicted in Fig. 1. The modelocked laser oscillator consists of a hemiconfocal resonator

Manuscript received July 23, 1982; revised November 12, 1982.

The authors are with the Naturwissenschaftliche Fakultät II-Physik, Universität Regensburg, 8400 Regensburg, Germany.

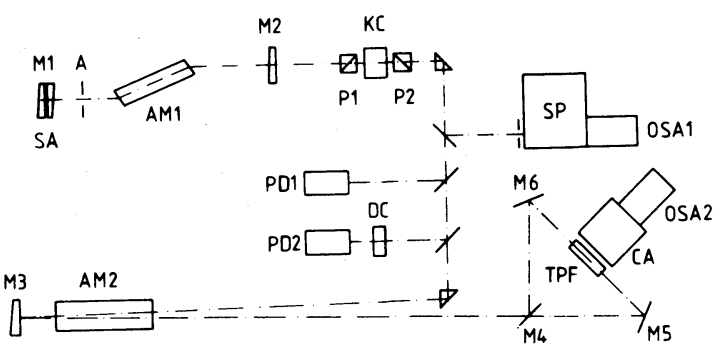

Fig. 1. Experimental setup. $M 1-M 6$-mirrors; $S A$-mode-locking dye cell; $A$-aperture; $A M 1$ and $A M 2$-active media; $P 1, P 2$-polarizers; $K C$-Kerr cell; $S P$-spectrograph; OSA 1 and OSA2-optical multichannel analyzers; $D C$-saturable absorber cell for intensity detection; $P D 1$ and PD2-photodetectors; TPF-two-photon fluorescence cell for pulse duration measurement; $C A$-camera.

with a plane 100 percent mirror $M 1$ and an output mirror $M 2$ of 30 percent reflectivity and $3 \mathrm{~m}$ curvature. The saturable absorber cell $S A$ is contacted to the front mirror $M 1$ (cell thickness $0.1 \mathrm{~mm}$, absorber Eastman 9860). Nd-phosphate, $\mathrm{Nd}$-fluorophosphate, and $\mathrm{Nd}$-silicate glass rods of $l=13 \mathrm{~cm}$ length and $d=9 \mathrm{~mm}$ diameter (Schott LG 703, LG 802, and LG 630, see Table I) are used as active media $A M 1$.

Single picosecond pulses are selected from the pulse train at varying positions with a spark-gap-triggered Kerr cell system $K C$ [19]. In some experiments, for pulse selection towards the end of the pulse train, a krytron-triggered system of two Pockel's cells in series was used [20].

The peak intensity of the light pulses is determined by a saturable absorber technique [21] with dye cell $D C$ and photodetectors $P D 1$ and $P D 2$. The spectra of the picosecond pulses are measured with either a $60 \mathrm{~cm}$ grating spectrograph (1200 $1 / \mathrm{mm}$, spatial dispersion $0.9 \mathrm{~nm} / \mathrm{mm}$ ) or a $25 \mathrm{~cm}$ spectrometer $(1200 \mathrm{l} / \mathrm{mm}$, dispersion $2.8 \mathrm{~nm} / \mathrm{mm})$. The spectra are detected with an optical spectrum analyzer OSA 1 (silicon vidicon). The pulse durations are determined by the two-photon fluorescence technique [22] (dye $2.5 \times 10^{-3}$ molar rhodamine $6 \mathrm{G}$ in ethanol). For this purpose, the pulses are increased in energy by double passage through a laser amplifier $A M 2$.

\section{RESUlts}

A typical pulse train is shown in Fig. 2. The peak intensity of the laser pulses at the first pulse train maximum is $I_{O, \max } \simeq$ $(4 \pm 2) \times 10^{9} \mathrm{~W} / \mathrm{cm}^{2}$ for a saturable dye transmission of $T_{0}=$ 85 percent (single pass) and an output mirror reflectivity of $R=$ 30 percent. This intensity value occurs inside the laser oscillator at the position of the active medium. The peak intensities $I_{O, \max }$ are approximately the same for all three investigated Nd-glass types. They are limited by two-photon absorption 
TABLE I

InVestigated Laser Glass Rods (Data from Schott Data SheETS)

\begin{tabular}{|c|c|c|c|c|c|c|}
\hline Type & $\begin{array}{l}\text { Peak } \\
\text { Wavelength } \\
\qquad \begin{array}{c}\lambda \\
{[\mathrm{nm}]}\end{array}\end{array}$ & $\begin{array}{c}\mathrm{Nd}^{3+} \text {-Con- } \\
\text { centration } \\
\mathrm{C} \\
{\left[\mathrm{cm}^{-3}\right]}\end{array}$ & $\begin{array}{l}\text { Inhomogeneous } \\
\text { halfwidth } \\
\Delta \widetilde{v}_{\text {inh }} \\
{\left[\mathrm{cm}^{-1}\right]}\end{array}$ & $\begin{array}{l}\text { Fluorescence } \\
\text { lifetime } \\
{ }^{\tau} \mathrm{Fl} \\
{[\mu \mathrm{s}]}\end{array}$ & $\begin{array}{l}\text { Emission } \\
\text { cross-section } \\
\sigma_{\mathrm{em}} \\
{\left[\mathrm{cm}^{2}\right]}\end{array}$ & $\begin{array}{l}\text { Nonlinear } \\
\text { refractive index } \\
n_{2} \\
\text { [esu] }\end{array}$ \\
\hline $\begin{array}{l}\text { Schott LG } 703 \\
\text { (phosphate) }\end{array}$ & 1053 & $2.82 \times 10^{20}$ & 189 & 240 & $4.1 \times 10^{-20}$ & $1.08 \times 10^{-13}$ \\
\hline $\begin{array}{l}\text { Schott LG } 802 \\
\text { (fluorcphos- } \\
\text { phate) }\end{array}$ & 1052 & $2.86 \times 10^{20}$ & 198 & 380 & $2.7 \times 10^{-20}$ & $6.3 \times 10^{-14}$ \\
\hline $\begin{array}{l}\text { Schott LG } 630 \\
\text { (silicate) }\end{array}$ & 1058 & $2.78 \times 10^{20}$ & 197 & 640 & $1.1 \times 10^{-20}$ & $1.4 \times 10^{-13}$ \\
\hline
\end{tabular}

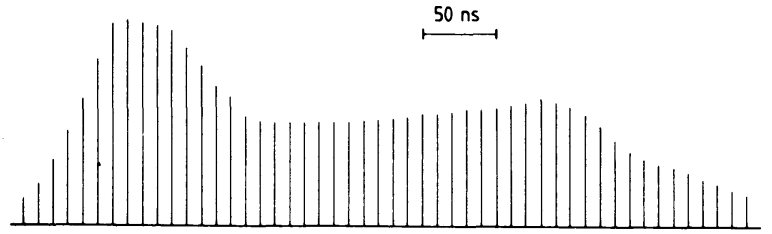

Fig. 2. Typical oscilloscope trace of a mode-locked pulse train. Laser glass-Schott LG 703 (phosphate); small signal dye transmission $T_{o}=$ 0.85 (single pass).

(a)

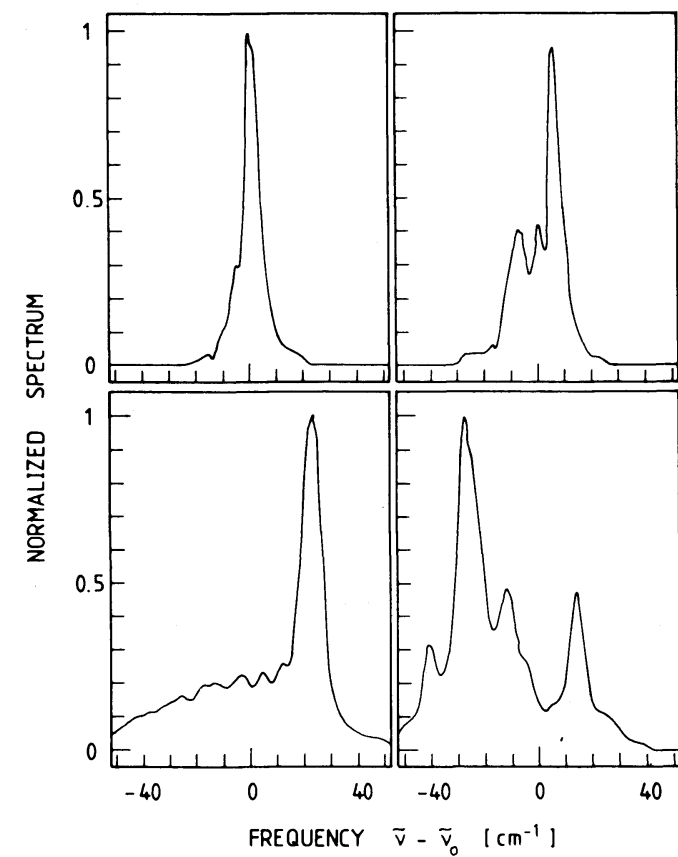

Fig. 3. Spectra of single picosecond laser pulses of mode-locked Ndphosphate glass laser (Schott LG 703). (a) Switching position-7 pulses before first train maximum $(j=-7)$; height $h=0.26$ of height at maximum; duration-bandwidth product $\Delta v \Delta t \simeq 1$ (FWHM). (b) $j=-3, h=0.7, \Delta \nu \Delta t \simeq 1$. (c) $j=6, h \simeq 0.65 ; \Delta \nu \Delta t \simeq 1.8$. (d) $j=$ 31 (second train maximum), $h \simeq 0.55, \Delta \nu \Delta t \simeq 2$.

in the active medium [17]-[18]. The durations of the light signals are found to be in the range of $\Delta t=6 \pm 3$ ps (FWHM) for all investigated laser glasses. They are approximately constant along the pulse train.

Spectra of single picosecond pulses selected at different positions along the pulse train are depicted in Figs. 3-5 for $\mathrm{Nd}-$ (a)

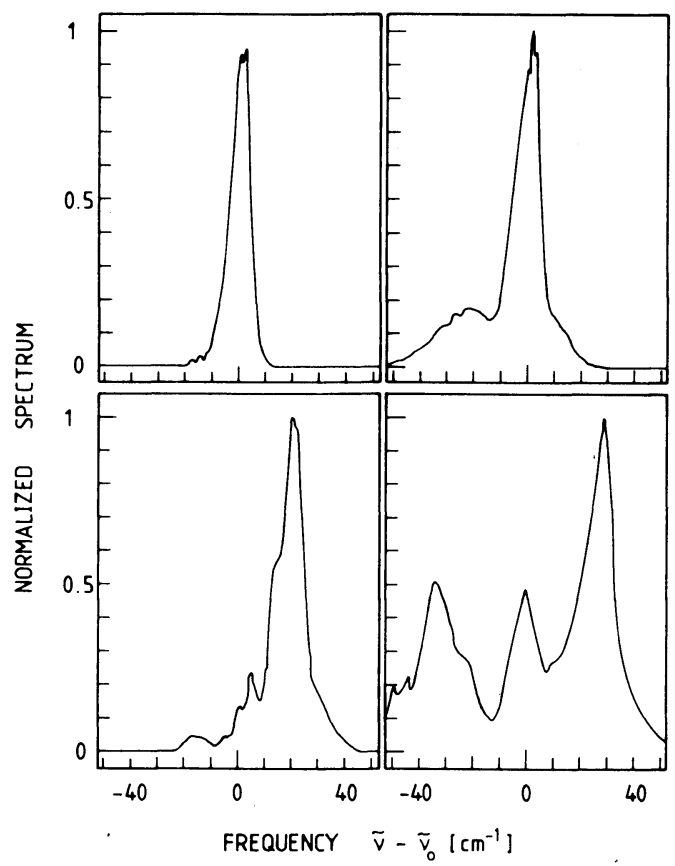

(b)

(d)

Fig. 4. Picosecond pulse spectra with Nd-fluorophosphate laser rod (Schott LG 702). (a) Switching position $j=-12$, switching height $h=0.15, \Delta \nu \Delta t \simeq 1$. (b) $j=-3, h=0.8, \Delta \nu \Delta t \simeq 1.9$. (c) $j=14$, $h=0.5, \Delta \nu \Delta t \simeq 2.2$. (d) $j=13$ (second train maximum), $h=0.8$, $\Delta \nu \Delta t \simeq 2.6$.

phosphate, Nd-fluorophosphate, and $\mathrm{Nd}$-silicate glass rods, respectively. Figs. 3(a), 4(a), and 5(a) are obtained for pulses of the rising part, approximately a factor of five below the pulse train maximum. Figs. 3(b), 4(b), and 5(b) represent spectra around the first train maximum. Figs. 3(c), 4(c), and 5(c) belong to the trailing part behind the first train maximum. The spectra of Figs. 3(d) and 4(d) are taken around the second pulse train maximum. The spectrum of Fig. 5(d) is selected at about the same position as the spectrum of Fig. 5(c) to indicate the shot-to-shot variation in the case of $\mathrm{Nd}$-silicate glass.

The single picosecond pulse spectra show the following behavior along the pulse train. 1) in the early part of the train where the pulse heights are less than 0.15 , the maximum height the signals are nearly bandwidth limited [Figs. 3(a), 4(a), and 5(a)] . 2) Up to the first pulse train maximum, the halfwidth of the spectra increases, broad wings become detectable, and 


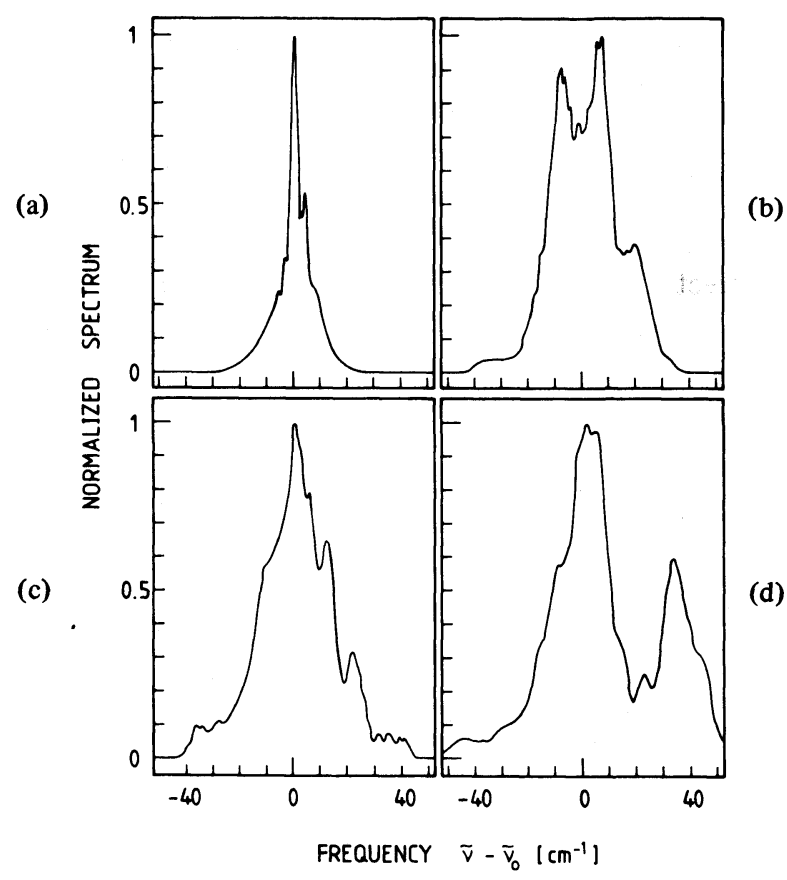

Fig. 5. Pulse spectra of mode-locked Nd-silicate glass laser (Schott LG 630). (a) Switching position $j=-17$, switching height $h=0.17$, $\Delta \nu \Delta t \simeq 0.6$. (b) $j=-1, h=0.95, \Delta \nu \Delta t \simeq 4.3$. (c) $j=8, h=0.8$ $\Delta \nu \Delta t \simeq 5$. (d) $j=9, h=0.4, \Delta \nu \Delta t \simeq 8.8$.

the spectral distribution becomes modulated [Fig. 3(b), 4(b), and 5(b)]. The spectra are asymmetric; they extend more strongly to the low-frequency side, while the spectral peak position slightly shifts to the high-frequency side. This behavior is expected by self-phase modulation of temporal asymmetric pulses with steeper rising than decaying parts [23]-[25], as is the case for our pulses [26], [27]. 3) In the trailing part behind the first train maximum, the spectral emission at the central frequency is reduced and a new small component $\left(\Delta \tilde{\nu} \simeq 10 \mathrm{~cm}^{-1}\right)$ builds up at the high-frequency side [Figs. $3(\mathrm{c})$ and $4(\mathrm{c})]$. Its distance from the central frequency is $\delta \tilde{\nu} \simeq 25 \pm 5 \mathrm{~cm}^{-1}$. 4) Around the second pulse train maximum, the spectrum frequently has a peak at the low-frequency side [Figs. 3(d) and 4(d)] .

The buildup of intense spectral components at the wings is less pronounced in the $\mathrm{Nd}$-silicate laser. This glass type has the highest nonlinear refractive index. The self-phase modulation broadens the spectra already early in the pulse trains, and acts against spectral hole burning.

The effects of self-phase modulation on the spectral development are investigated with $\mathrm{CS}_{2}$ samples in the laser oscillator. The nonlinear refractivity of $\mathrm{CS}_{2}$ is about a factor of 100 greater than that of laser glass $\left(\mathrm{n}_{2}\left(\mathrm{CS}_{2}\right)=1.1 \times 10^{-11} \mathrm{ESU}=1.22 \mathrm{X}\right.$ $10^{-20} \mathrm{~m}^{2} / \mathrm{V}^{2}$ [28], for response times of contributions to $n_{2}$ of $\mathrm{CS}_{2}$, see [29], [30], [44]). The effects of self-phase modulation are strongly enhanced by the huge nonlinear refractivity of $\mathrm{CS}_{2}$. In Figs. 6(a)-(d), spectra at the pulse train maximum are shown for $\mathrm{CS}_{2}$ samples in the resonator with lengths of $0.5,1,2$, and $5 \mathrm{~cm}$, respectively. The Nd-phosphate laser rod LG 703 is used as the active medium. The spectral structuring increases with $\mathrm{CS}_{2}$ cell length from Fig. $\left.6(\mathrm{a})(0.5 \mathrm{~cm} \mathrm{CS})_{2}\right)$ to Fig. 6(d) $\left(5 \mathrm{~cm} \mathrm{CS}_{2}\right)$. Spectra obtained with 2 and $5 \mathrm{~cm} \mathrm{CS}_{2}$ samples were similar, i.e., the spectral shapes shown in Fig. 6(c) $\left.(2 \mathrm{~cm} \mathrm{CS})_{2}\right)$ are also found for $5 \mathrm{~cm} \mathrm{CS}_{2}$ samples, and vice versa. The spectral shapes are determined by self-phase modulation. (a)

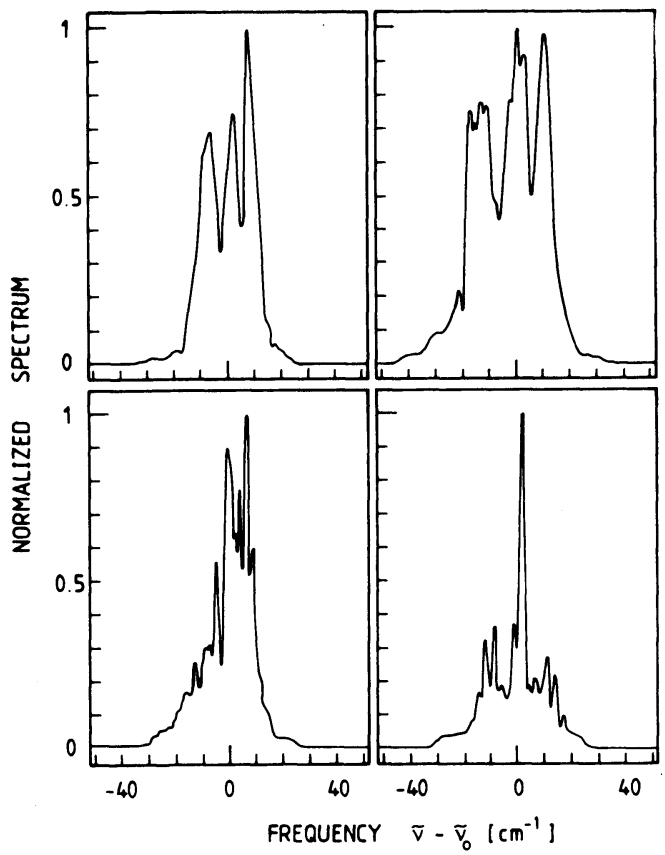

(b)

(d)

Fig. 6. Pulse spectra of mode-locked Nd-phosphate laser with $\mathrm{CS}_{2}$ samples in resonator. (a) Cell length $l=0.5 \mathrm{~cm}$, switching position $j=$ -3 , switching height $h=0.6, \Delta \nu \Delta t \simeq 4$. (b) $l=1 \mathrm{~cm}, j=-1, h=0.9$, $\Delta \nu \Delta t \simeq 6$. (c) $l=2 \mathrm{~cm}, j=0, h=1, \Delta \nu \Delta t \simeq 2.7$. (d) $l=5 \mathrm{~cm}, j=$ $-1, h=0.9, \Delta \nu \Delta t \simeq 0.5$.

Contributions to the spectra from spectral hole burning are not observable. Towards the end of the pulse train, broad modulated side peaks (height $\lesssim 0.4$ height of central peak) are sometimes observed. Second pulse train maxima rarely occur.

\section{Discussion}

The spectral development along the pulse train may be explained by taking into account the spectral hole burning in the inhomogeneously broadened active medium [31]-[39] in addition to self-phase modulation.

The inhomogeneous width of the neodymium laser transition is about $\Delta \tilde{\nu}_{\text {inh }} \simeq 200 \mathrm{~cm}^{-1}$ (FWHM, Table I), while the homogeneous width is approximately $20 \mathrm{~cm}^{-1}$ [32], [34], [35], [37], [39]. A refilling of the hole within the duration of the pulse train does not take place since the cross-relaxation time $T_{3}$ is in the $10^{-5}-10^{-4} \mathrm{~s}$ region. Values of $T_{3}=(70 \pm 20) \mu \mathrm{s}$ for phosphate glass and $T_{3}=(200 \pm 50) \mu \mathrm{s}$ for silicate glass are reported in [40]. Similar data were found in [35]. Our measurements with the free-running $\mathrm{Nd}$-glass laser (without saturable absorber) indicated a cross-relaxation time $T_{3}>5 \mu \mathrm{s}$. At the laser threshold, a single spike was emitted with spectral width $\lesssim 4 \mathrm{~cm}^{-1}$. Operating the laser with a pump energy of a factor of five above threshold, an irregular train of spikes was emitted with a distance between successive spikes of about 5 $\mu \mathrm{s}$. In this case, the spectral emission broadened considerably and built up large wings.

The picosecond pulse evolution in the passive mode-locking process may be divided into 1) a prelaser region (end at first laser threshold), 2) a linear region (end at second laser threshold), 3) a nonlinear phase without gain saturation, and 4) a nonlinear phase coinciding with saturation phenomena (for reviews see [8], [41]).

1) and 2): In the prelaser and linear regions, the spectrum is 
narrowed by natural mode selection. Light amplification occurs only in a narrow spectral range at the maximum of the gain profile since the laser is operated only slightly above threshold. the statistical spontaneous emission is amplified in the linear phase.

3): The nonlinear phase begins when the strongest fluctuation spike is intense enough to reduce the absorption of the saturable dye. This spike is preferably amplified, due to its reduced losses. During the opening time of the absorber, the laser loss is reduced for all frequency components of the emission (ground state of absorber is depopulated) and strong amplification takes place over a wide frequency range. As long as saturation effects do not occur, the spectral half-width remains small, since amplification is largest at the center of the gain profile.

4): Towards the first pulse train maximum, the central frequency components of the generated emission deplete the inversion of the inhomogeneously broadened laser medium and a hole is burned in the center of the gain profile (hole formation in the inversion already begins when pulse heights are a factor of 50 below pulse height at train maximum [42]). Beyond the pulse train maximum, the effective amplification factor at the center frequency drops below one, and the height of the central peak reduces while the wings are strongly amplified during the opening period of the absorber. The emission maximum appears at the high-frequency side (shift $\left.\delta \tilde{\nu} \simeq \Delta \tilde{\nu}_{\text {hom }}\right)$, where the temporal trailing part of the self-phase modulated pulse occurs. At this high-frequency side, the spectral extension by self-phase modulation is small and the inversion outside the central homogeneous width is not reduced by selfphase modulation up to the first pulse train maximum [see Figs. 3(b) and 4(b)]. At the low-frequency side, which corresponds to the temporal rising part of the self-phase modulated pulse, the spectral wings already extend beyond the central homogeneous linewidth around the first train maximum. The inversion in the low-frequency wing is reduced by the selfphase modulation. This is thought to be responsible for the asymmetric appearance of a spectral peak at the high-frequency side. Around the frequently occurring second pulse train maximum, a spectral peak is often observed at the low-frequency side [see Figs. 3(d) and 4(d)] .

The spectral peaks at the high- and low-frequency sides are formed by amplification of statistically emitted fluorescence light during the opening time of the absorber in the nonlinear phase. This spectral fluorescence component can build up at the decaying part of the bleaching pulse, where the saturable absorber is transparent and the two-photon absorption is weak (small pulse intensity).

The two-photon absorption of laser light in the Nd-glass rod limits the maximum pulse intensity since it introduces an additional loss. An estimate of the two-photon absorption coefficient is obtained by equating the two-photon absorption loss and the dye bleaching gain, i.e.,

$$
T_{\mathrm{TPA}}=\frac{1}{1+\alpha^{(2)} I_{O} l}=T_{o}
$$

where $T_{O}$ is the small signal dye transmission (single pass), $T_{\text {TPA }}$ is the two-photon transmission, $l$ is the length of the glass rod, $I_{O}$ is the laser peak intensity, and $\alpha^{(2)}$ is the twophoton absorption coefficient. A value of $\alpha^{(2)} \simeq 3 \times 10^{-12}$ $\mathrm{cm} / \mathrm{W}$ is obtained for our experimental parameters $\left(I_{O}=4 \times\right.$ $\left.10^{9} \mathrm{~W} / \mathrm{cm}^{2}, l=13 \mathrm{~cm}, T_{O}=0.85\right)$.

The effect of the nonlinear refractive index $n_{2}$ of the active media on self-focusing is estimated for our experimental conditions (Nd-phosphate glass). An expression for the whole beam self-focusing length is [45], [46]

$$
z_{f}=\frac{k w^{2}}{2}\left(\frac{P}{P_{c}}-1\right)^{-1 / 2}
$$

where $k=2 \pi / \lambda$ is the wave number of laser light; $w=w_{o}$ $\left(1+4 z^{2} / b^{2}\right)^{1 / 2}$ is the spot size of the laser beam at the active medium; $P=I_{O} w^{2} / 4$ is the laser power and $P_{c}=\lambda^{2} c /\left(32 \pi^{2} n_{2}\right)$ is the critical power. $w_{o}=(\lambda b / 2 \pi)^{1 / 2}$ is the minimum spot size in the laser resonator. $b$ is the curvature of the output mirror (front mirror is plane in our case). $z$ is the distance from the plane front mirror. For our laser data of $\lambda=1.055 \mu \mathrm{m}, z=90$ $\mathrm{cm}, b=3 \mathrm{~m}, n_{2}=1.08 \times 10^{-13} \mathrm{ESU}$, and $I_{O L}=4 \times 10^{9} \mathrm{~W} /$ $\mathrm{cm}^{2}$, we find $z_{f}=83 \mathrm{~cm}$. The length of the active medium of $l=13 \mathrm{~cm}$ is considerably shorter than the self-focusing length. The nonlinear refractive index $n_{2}$ of the active medium slightly changes the wavefront curvature in the resonator and the laser oscillator may be described by a resonator with slightly changed curvature of the mirrors.

The spectral development of picosecond pulses along the pulse train of a mode-locked Nd-glass laser was simulated by computer calculations. A complete description of this theoretical analysis will be given elsewhere [42]. Some preliminary results are presented in Figs. 7 and 8 which confirm the model of spectral pulse development described above.

In Fig. 7, the spectral development of a picosecond pulse train is depicted in the case of absence of self-phase modulation. Curve 1 shows the spectral distribution of spontaneous emission. Curve 2 shows a spectral shape at the end of the prelaser region, while curve 3 shows the spectrum at the end of the linear phase (natural mode selection). The spectrum at the first pulse train maximum is shown in curve 4 . Curve 5 shows the spectral distribution 40 pulses after the pulse train maximum. The spectral shape at the second pulse train maximum (70 pulses after the first train maximum) is depicted by curve 6 . The buildup of the spectral wings due to spectral hole formation in the gain profile and light amplification within the opening time of the absorber is clearly seen in curves 4-6. Parameters used in the calculations are listed in the figure captions. The inclusion of two-photon absorption reduces the absolute height of spectral curves 4-6, but has no influence on the gross spectral development of the pulse train.

The spectral development of a pulse train due to self-phase modulation is depicted in Fig. 8. In these calculations, it is assumed that no spectral hole burning in the gain profile occurs. A Gaussian gain profile corresponding to the spontaneous fluorescence emission is used in the calculations. The pulse parameters are adjusted to experimental pulse train data of Fig. 2. In 


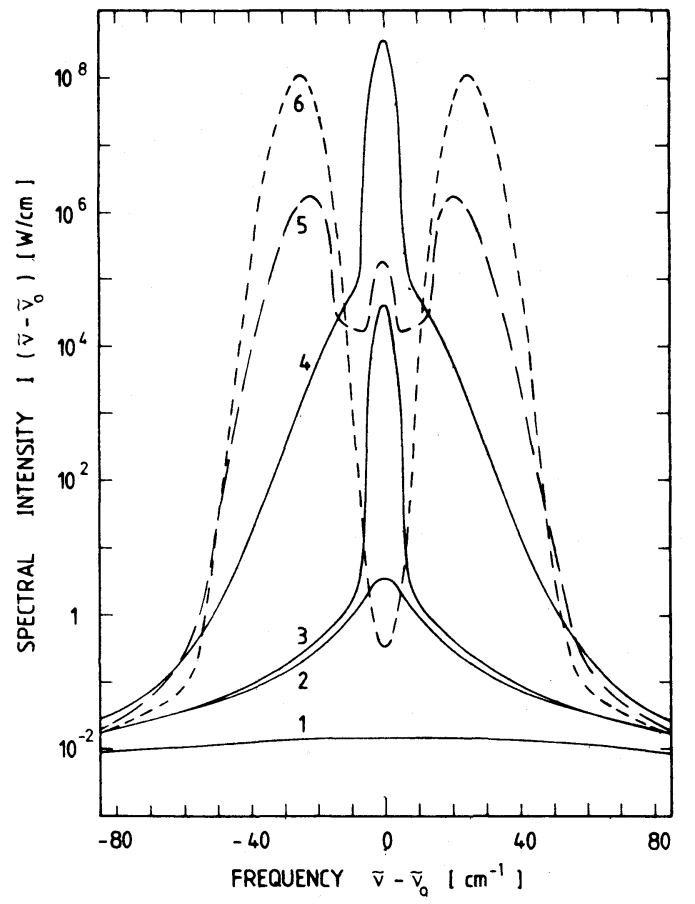

Fig. 7. Calculated spectral development of a picosecond pulse train. Effects of self-phase modulation are not included $\left(n_{2}=0\right)$. Curve 1spontaneous emission; curve 2-end of prelaser region; curve 3-end of linear region; curve 4-first pulse train maximum; curve 5-40 pulses after first maximum; curve 6 -at second pulse train maximum $(70$ pulses after first maximum). Data used in the calculation-amplification per roundtrip in linear phase is 1.05 ; small signal absorber transmission $T_{o}=0.85$ (single pass); output mirror reflectivity is 0.3 ; additional losses in resonator per roundtrip are 0.1 ; active medium is $\mathrm{Nd}$ phosphate glass with data of Table I; two-photon absorption cross section $\alpha^{(2)}=1.5 \times 10^{-12} \mathrm{~cm} / \mathrm{W}$ (does not influence gross features of the picture). Pulse duration $\Delta t=4 \mathrm{ps}$. Obtained peak pulse intensity $I_{O}=5.4 \times 10^{9} \mathrm{~W} / \mathrm{cm}^{2}$. For a detailed discussion, see $[42]$.

particular, the experimentally observed asymmetric temporal pulse shape [26], [27], which leads to asymmetric spectra, is included in the calculations. Fig. 8(a) shows the spectrum of the fourth pulse of Fig. 2. The spectrum is nearly bandwidth limited. Fig. 8(b) presents a spectrum at the pulse train maximum. It is broadened, modulated, and asymmetric. The spectrum of Fig. 8(c) is taken from a pulse in the trailing part of the pulse train (10 pulses behind first train maximum of Fig. 2). The spectrum is further broadened and modulated, and the asymmetric shape remains.

The spectra of Fig. 8 are not able to explain the experimental findings in Figs. 3(c), 3(d), 4(c), and 4(d). These spectra may be understood by combined effects of self-phase modulation and spectral hole burning.

\section{Conclusions}

The spectral development of picosecond light pulses along the pulse train of a mode-locked $\mathrm{Nd}$-glass laser was studied. The spectral behavior beyond the pulse train maximum is explained by simultaneous action of self-phase modulation and spectral hole burning in the gain profile of the active medium. The position of spectral peaks at frequencies shifted from the central

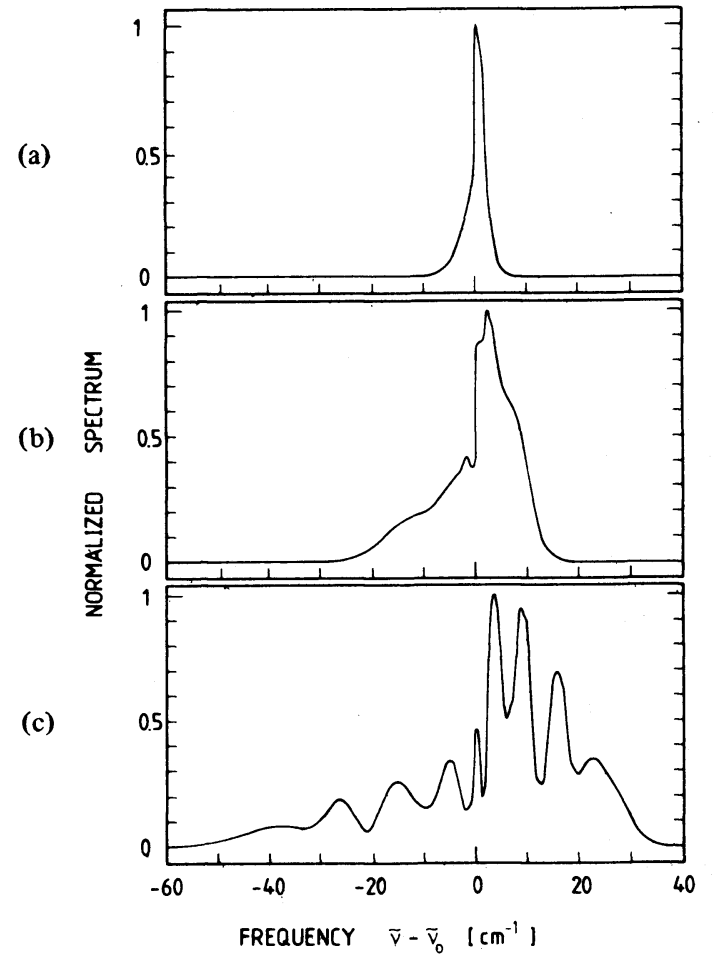

Fig. 8. Calculated spectral shape of picosecond pulses along pulse train. Effects of spectral hole burning are not included. For the shape of the pulse train, see Fig. 2. Peak intensity at train maximum $I_{O, \max }=$ $7 \times 10^{9} \mathrm{~W} / \mathrm{cm}^{2}$. Shape of first pulse of train $s(r, t)=\exp \left[-\left(r / r_{o}\right)^{2}\right]$ $\left\{\exp \left[-\left(t / t_{o}\right)^{2}\right][1-\theta(t)]+\cosh ^{-2}\left(\kappa t / t_{o}\right) \theta(t)\right\} . \quad t_{o}=\Delta t 2^{-1}(\ln$ $2)^{-1 / 2}=3 \mathrm{ps}, k=1.6$. Spectral gain profile $g\left(\tilde{\nu}-\tilde{\nu}_{o}\right)=\exp [-(\tilde{\nu}-$ $\left.\left.\tilde{\nu}_{O}\right)^{2} / \tilde{\nu}_{i n h}^{2}\right] ; \tilde{\nu}_{i n h}=\Delta \tilde{\nu}_{i n h} 2^{-1}(\ln 2)^{-1 / 2}=113 \mathrm{~cm}^{-1}$. Spectra are integrated over spatial cross section. (a) Pulse at position $j=-4$ (4 pulses before maximum). (b) Pulse at train maximum $j=0$. (c) Pulse at at position $j=10$ (trailing part of train).

frequency gives an estimate of the homogeneous linewidths of the active media. The two-photon absorption in the Nd-glass rods limits the peak pulse intensity in the train maxima. The two-photon absorption cross sections may be estimated from the measured peak intensities.

The interpretation of the spectral development of picosecond pulses along the pulse train of a mode-locked $\mathrm{Nd}$-glass laser is in agreement with computer simulations [42].

The development of $\mathrm{Nd}$-glass rods with enlarged homogeneous linewidths [34], [43] allows the energy extraction of inversion over a wider frequency range and hinders the spectrally selective gain depletion.

Insertion of a spectral narrowing solid etalon into the resonator hinders the buildup of spectral side peaks and the formation of second pulse train maxima [47], [48]. The effects of spectral hole burning on the spectral pulse shapes are suppressed by the spectral transmission profile of the etalons.

\section{ACKNOWLEDGMENT}

The authors are indebted to Prof. M. Maier for stimulating discussions. They thank T. Ascherl for technical assistance. They are also grateful to Dr. N. Neuroth of Schott Glaswerke, Mainz, Germany, for the Nd-silicate glass rod. 


\section{REFERENCES}

[1] R. C. Eckardt, J. N. Bradford, and C. H. Lee, "Temporal and spectral development of mode-locking in a ring cavity Nd:glass laser," Appl. Phys. Lett., vol. 19, pp. 420-423, Nov. 1971.

[2] D. von der Linde, "Experimental study of single picosecond light pulses," IEEE J. Quantum Electron., vol. QE-8, pp. 328-338, Mar. 1972.

[3] R. C. Eckardt, C. H. Lee, and J. N. Bradford, "Effect of self-phase modulation on the evolution of picosecond pulses in a Nd:glass laser," Opto-Electron., vol. 6, pp. 67-85, Jan. 1974.

[4] A. B. Mirnov and O. B. Shatberashvili, "Structure of the spectrum of a single ultrashort pulse emitted by a neodymium-glass laser," Sov. J. Quantum Electron., vol. 4, pp. 805-806, Dec. 1974.

[5] D. J. Bradley and W. Sibbett, "Streak-camera studies of picosecond pulses from a mode-locked Nd:glass laser," Opt. Commun., vol. 9, pp. 17-20, Sept. 1973.

[6] W. Zinth, A. Laubereau, and W. Kaiser, "Generation of chirpfree picosecond pulses," Opt. Commun., vol. 22, pp. 161-164, Aug. 1977.

[7] T. R. Royt, "Passive mode-locking of the Nd-glass oscillator at high repetition rate with thermally compensated phosphate glasses," Opt. Commun., vol. 35, pp. 271-276, Nov. 1980.

[8] W. H. Lowdermilk, "Technology of bandwidth-limited ultrashort pulse generation," in Laser Handbook, Vol. 3, M. L. Stitch, Ed. Amsterdam, The Netherlands: North Holland, 1979, pp. 361420.

[9] M. A. Duguay, J. W. Hansen, and S. L. Shapiro, "Study of the $\mathrm{Nd}$ : glass laser radiation," IEEE J. Quantum Electron., vol. QE-6, pp. 725-743, Nov. 1970.

[10] V. V. Korobkin, A. A. Malyutin, and A. M. Prokhorov, "Phase self-modulation and self-focusing of neodymium laser radiation with mode-locking," JETP Lett., vol. 12, pp. 150-152, Sept. 1970.

[11] G. R. Fleming, I. R. Harrowfield, A.E.W. Knight, J. M. Morris, R. J. Robbins, and G. W. Robinson, "Properties of single picosecond pulses from neodymium: phosphate glass," Opt. Commun., vol. 20, pp. 36-41, Jan. 1977.

[12] J. R. Taylor, W. Sibbett, and A. J. Cormier, "Bandwidth-limited picosecond pulses from a neodymium phosphate glass oscillator," Appl. Phys. Lett., vol. 31, pp. 184-186, Aug. 1977.

[13] G. Dikchyus, E. Zhilinskas, A. Piskarskas, and V. Sirutkaitis, "Statistical properties and stabilization of a picosecond phosphateglass laser with $2 \mathrm{~Hz}$ repetition frequency," Sov. J. Quantum Electron., vol. 9, pp. 950-955, Aug. 1979.

[14] R. C. Eckardt, "Self-focusing in mode-locked neodymium: glass laser oscillators," IEEE J. Quantum Electron., vol. QE-10, pp.4856, Jan. 1974.

[15] R. R. Cubeddu and O. Svelto, "Theory of laser self-locking in the presence of host dispersion," IEEE J. Quantum Electron., vol. QE-5, pp. 495-502, Oct. 1969.

[16] W. E. Lamb, "Theory of an optical maser," Phys. Rev., vol. 134A, pp. 1429-1450, June 1964.

[17] A. Penzkofer and W. Kaiser, "Nonlinear loss in Nd-doped laser glass," Appl. Phys. Lett., vol. 21, pp. 427-430, Nov. 1972.

[18] A. Caruso, R. Gratton, and W. Seka, "Mode-locked ring-laser and ring-amplifier characteristics," IEEE J. Quantum Electron., vol. QE-9, pp. 1039-1043, Nov. 1973.

[19] D. von der Linde, O. Bernecker, and A. Laubereau, "A fast electrooptic shutter for the selection of single picosecond laser pulses," Opt. Commun., vol. 2, pp. 215-218, Oct. 1970.

[20] J. Biebl and A. Penzkofer, "Gating of optical multichannel analysers with krytron switches," J. Phys. Earth: Sci. Instrum., vol. 13, pp. 1328-1330, Dec. 1980.

[21] A. Penzkofer, D. von der Linde, and A. Laubereau, "The intensity of short light pulses determined with saturable absorbers," Opt. Commun., vol. 4, pp. 377-379, Jan. 1972.

[22] J. A. Giordmaine, P. M. Rentzepis, S. L. Shapiro, and K. W. Wecht, "Two-photon excitation of fluorescence by picosecond light pulses," Appl. Phys. Lett., vol. 11, pp. 216-218, Oct. 1967.

[23] T. K. Gustafson, J. P. Taran, H. A. Haus, J. R. Lifshitz, and P. L. Kelley, "Self-modulation, self-steepening, and spectral development of light in small-scale trapped filaments," Phys. Rev., vol. 177 , pp. 306-313, Jan. 1969.

[24] O. Svelto, "Self-focusing, self-trapping, and self-phase modulation of laser beams," in Progress in Optics, Vol. 12, E. Wolf, Ed. Amsterdam, The Netherlands: North-Holland, 1974, pp. 1-51.

[25] S. A. Akhmanov, R. V. Khokhlov, and A. P. Sukhorukov, "Self- focusing, self-defocusing and self-modulation of laser beams," in Laser Handbook; Vol. 2, F. T. Arrecchi and E. O. Schultz-Dubois, Eds. Amsterdam, The Netherlands: North-Holland, 1972, ch.E3, pp. 1151-1228.

[26] J. Wiedmann and A. Penzkofer, "Determination of the shape and duration of picosecond light pulses by bleaching of dyes," Opt. Commun., vol. 30, pp. 107-112, July 1979.

[27] W. Leupacher and A. Penzkofer, "Analysis of a mode-locked Ndglass laser by four-wave mixing," Appl. Phys., vol. B29, pp. 263267, Dec. 1982.

[28] J. M. Cherlow, T. T. Yang, and R. W. Hellwarth, "Nonlinear optical susceptibilities of solvents," IEEE J. Quantum Electron., vol. QE-12, pp. 644-646, Oct. 1976.

[29] J. Etchepare, G. Grillon, R. Astier, J. L. Martin, C. Bruneau, and A. Antonetti, "Time resolved measurement of nonlinear susceptibilities by optical Kerr effect," in Picosecond Phenomena III (Springer Series Chem. Phys.), Vol. 23, K. B. Eisenthal, R. M. Hochstrasser, W. Kaiser, and A. Laubereau, Eds. Berlin, Germany: Springer-Verlag, 1982, pp. 217-220.

[30] J. M. Halbout and C. L. Tang, "Time-resolved observation of the nonlinear refractive index of molecular liquids by femtosecond interferometry," Appl. Phys., vol. B28, pp. 144-145, June 1982.

[31] W. H. Keene and J. A. Weiss, "The time-resolved spectrum of a neodymium glass laser," Appl. Opt., vol. 3, pp. 545-547, Apr. 1964.

[32] E. Snitzer and C. G. Young, "Glass lasers," in Lasers, Vol. 2, A. K. Levine, Ed. New York: Marcel Dekker, 1968, ch. 2, pp. 191-256.

[33] C. Brecher, L. A. Riseberg, and M. J. Weber, "Variations in the transition probabilities and quantum efficiency of $\mathrm{Nd}^{3+}$ ions in ED-2 laser glass," Appl. Phys. Lett., vol. 30, pp. 475-478, May 1977.

[34] J. M. Pellegrino, W. M. Yen, and M. J. Weber, "Composition dependence of $\mathrm{Nd}^{3+}$ homogeneous linewidths in glasses," J. Appl. Phys., vol. 51, pp. 6332-6336, Dec. 1980.

[35] S. A. Brawer and M. J. Weber, "Observation of fluorescence line narrowing, hole burning, and ion-ion energy transfer in neodymium laser glass," Appl. Phys. Lett., vol. 35, pp. 31-33, July 1979.

[36] M. J. Weber, "Laser excited fluorescence spectroscopy in glass," in Laser Spectroscopy in Solids, Topics in Applied Physics, Vol. 49 , W. M. Yen and P. M. Selzer, Eds. Berlin, Germany: SpringerVerlag, 1981, pp. 189-239.

[37] V. I. Nikitin, M. S. Soskin, and A. I. Khizhnyak, "Uncorrelated inhomogeneous broadening as the cause for the narrow-band output of an $\mathrm{Nd}^{3+}$-activated phosphate glass laser," Sov. Tech. Phys. Lett., vol. 3, pp. 5-6, Jan. 1977.

[38] V. V. Antsiferov, A. V. Ghiner, N. M. Derjy, K. P. Komarov, and K. G. Folin, "Spectro-kinetic characteristics of Nd:glass laser and the rate of spatial migration of inversion," Opt. Commun., vol. 14, pp. 388-391, Aug. 1975.

[39] V. B. Kravchenko and Yu. P. Rudnitsku, "Phosphate laser glasses (review)," Sov. J. Quantum Electron., vol. 9, pp. 399-415, Apr. 1979.

[40] A. A. Mak, D. S. Prilezhaev, V. A. Serebryakov, and A. D. Starikov, "Measurement of relaxation rates in glasses activated with $\mathrm{Nd}^{3+}$ ions," Opt. Spectrosc., vol. 33, pp. 381-385, Oct. 1972.

[41] D. J. Bradley, "Methods of generation," in Ultrashort Light Pulses, Topics in Applied Physics, Vol. 18, S. L. Shapiro, Ed. Berlin, Germany: Springer-Verlag, 1977, pp. 18-81.

[42] A. Penzkofer, "Theoretical analysis of spectral development of picosecond pulses of mode-locked Nd-glass lasers," to be published.

[43] M. J. Weber, "Homogeneous line widths and energy extraction in laser glass," in Lawrence Livermore Nat. Lab. 1980 Laser Progr., Ann. Rep. UCRL-50021-80, vol. 1, L. W. Coleman and W. F. Krupke, Eds., pp. 2-335-2-338.

[44] B. I. Greene and R. C. Farrow, "Femtosecond Transient Birefringence in $\mathrm{CS}_{2}$," in Picosecond Phenomena III (Springer Series Chem. Phys.), Vol. 23, K. B. Eisenthal, R. M. Hochstrasser, W. Kaiser, and A. Laubereau, Eds. Berlin, Germany: Springer-Verlag, 1982, pp. 209-211.

[45] A. Yariv, Quantum Electronics, 2nd ed. New York: Wiley, 1975.

[46] W. Koechner, Solid-State Laser Engineering. Berlin, Germany: Springer-Verlag, 1976.

[47] L. S. Goldberg, P. E. Shoen, and M. J. Marrone, "Repetively pulsed mode-locked Nd: phosphate glass laser oscillator-amplifier system," Appl. Opt., vol. 21, pp. 1474-1477, Apr. 1982.

[48] F. Graf, C. Löw, and A. Penzkofer, unpublished results. 


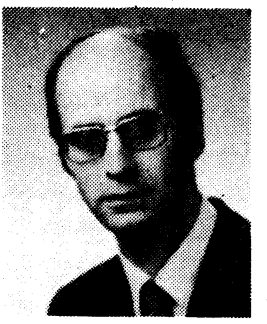

Alfons Penzkofer was born in Allersdorf, Germany, on October 19, 1942. He received the diploma, the Dr. rer. nat. and Dr.rer.nat.habil. degrees in physics from the Technical University of Munich, Munich, Germany, in 1971, 1974, and 1977 , respectively.

From 1970 to 1976 he worked in the Physics Department of the Technical University of Munich. Then he changed to the Naturwissenschaftliche Fakultät II-Physik at the University of Regensburg, Regensburg, Germany.

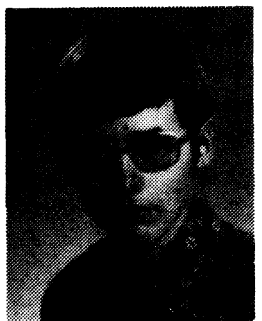

Norbert Weinhardt was born in Neuburg/Donau, Germany, on August 10, 1958. His study of physics and geography for the teaching profession began at the University of Regensburg, Regensburg, Germany, in 1977 . He is currently working towards his examination.

From 1981 to 1982 he was engaged in research on picosecond lasers at the Naturwissenschaftliche Fakultät II-Physik at the University of Regensburg. At both places he was involved in picosecond lasers and nonlinear optics. Since 1978 he has been Professor of Physics at the University of Regensburg. 